\title{
NUMERICAL ANALYSIS OF PRESS FITS AND BUSHING MIGRATION
}

\author{
Felipe José Vinaud ${ }^{1}$, Rodrigo Tadeu A. M. Troise ${ }^{1}$, Lucas Corrêa de Oliveira ${ }^{1}$, Marco
} Arisseto $^{1}$

${ }^{1}$ EMBRAER S.A., Avenida Brigadeiro Faria Lima, 2170, CEP 12297-901, São José dos Campos, São Paulo, Brazil (rodrigo.tadeu@embraer.com.br)

\begin{abstract}
Even though electronic devices are a reality in the aerospace industry, mechanical issues involving assembly through mechanical fit, more precisely press fits of bushings in primary structures and movable structures still demand attention in some aspects. Historically, this subject was demonstrated to affect all aircraft manufacturers during aircraft design, assembly and their operation. Also, existing methods in the literature are not capable of accurately predicting the interaction between the parts due to the combined behavior of differences in stiffness and the asymmetry of applied loads. This paper demonstrates a numerical methodology for evaluating press fits through a contact finite element model and takes into account considerations about materials compatibility, surface treatment and load asymmetries. Also a final solution considering dimensioning aspects and a comparison between the analytical and the numerical methodology are presented.
\end{abstract}

Keywords: Press fit, Second keyword, Non-linear contact model, Mechanical assembly.

\section{INTRODUCTION}

A bushing, also know as plain bearing, is a type of bearing composed by a bearing surface with no rolling elements, with the axle sliding over the bearing surface. This type of baring is the cheapest one in general. Its main features are low cost, low weight and high load capacity [Bruhn(1973)]. The present work is focused on one type of plain bearing: the bushing is an independent plain bearing inserted in a housing, commonly by means of cryogenic methods, when an interference fit is necessary to maintain bushing inside the housing. With respect to the bushing assembly, some concerns are raised, such as the correct temperature for freezing the bushing (cryogenics) and/or heat the housing hole, the bushing/ housing hole alignment during assembly and the correct corrosion inhibition compound to avoid corrosion between the parts. Material compatibility is also a concern, since an erroneous material combination between bushing/housing and axle can lead to premature wear and corrosion. A suitable geometry must be defined by the designer, to ensure the intended function to the bushing, avoiding undesired effects such as bushing migration and play inside the housing, what could lead to reduced fatigue life of the components. The existing analytical methodologies are too restrictive, enabling to evaluate the bushing behavior only when the external load 
from the axle is imposed in axial or radial directions. Based on this, the present work intends to present a comparison study between analytical methods and newcomer FEM techniques, demonstrating the differences between them and the capabilities of this new engineering tool, allowing for the analysis of assymmetrical and bending loads.

\section{MATERIAL COMPATIBILITY AND ROUGHNESS}

Bushings must be made with durable, low friction and low wear materials that, when in contact with the axle, will sustain high loads under special conditions such as low and high temperatures and corrosive environments. An erroneous material choice can lead to corrosion of the bushing, housing and axle. Also, the choice of the best bushing material to avoid corrosion of the housing and axle does not necessarily mean a guarantee of durability of the bushing [Ashby and Jones(2011)].

The use of the same material for housing, bushing, and axle should be avoided, since it is preferable to change a cheap component (i.e. the bushing) instead of the housing or axle which are more expensive. Thus, a softer bushing, can be replaced whenever it has worn too much.

In some cases, the correct roughness specification of the parts, which is directly associated to premature wear, is not enough to avoid the wear. With that in mind, some wear prevention actions should be addressed, such as periodic grease application or the use of a bi-material combination, with a metal bushing core and a plastic bearing surface. A proper choice of contact parameters will reduce friction and, consequently, wear.

\section{THEORETICAL ANALYSIS}

\subsection{Interference fit retention load capability}

Nowadays, the bushing-housing retention load can easily be calculated, based on the fact that when two cylindrical parts are assembled by shrinking or press fitting, a contact pressure is created between the two parts [Budynas and Nisbett(2008)]. In a shrink fit, the outer radius of the bushing is larger than the inner radius of the housing. After assembly, the interference contact pressure generates a radial stress, with the opposite sign of the pressure. The radial pressure is given by:

$$
p=\frac{\delta}{R\left[\frac{1}{E_{0}}\left(\frac{r_{0}^{2}+R^{2}}{r_{0}^{2}-R^{2}}+\nu_{0}\right)+\frac{1}{E_{i}}\left(\frac{R^{2}+r_{i}^{2}}{R^{2}-r_{i}^{2}}+\nu_{i}\right)\right]}
$$

The notation used in equation 1 is shown in Figure 1 


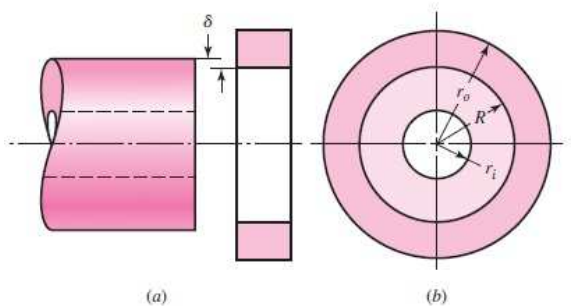

Figure 1. Notation for press and shrink fits [Budynas and Nisbett(2008)]

In order to obtain the interference fit retention load capability $F_{\text {retention }}$, it is necessary to multiply the pressure $p$ by the friction coefficient between lug and bushing materials and also by the contact area (with length $L$ ), as follows:

$$
F_{\text {retention }}=\mu_{\text {out }} 2 \pi R L p=\mu_{\text {out }} \pi D L p
$$

\subsection{Force to remove the bushing due to friction coupling between bushing and axle}

The loads imposed to the bushing by the axle tends to induce a pressure distribution along the half of the internal diameter of the bushing, as shown below:

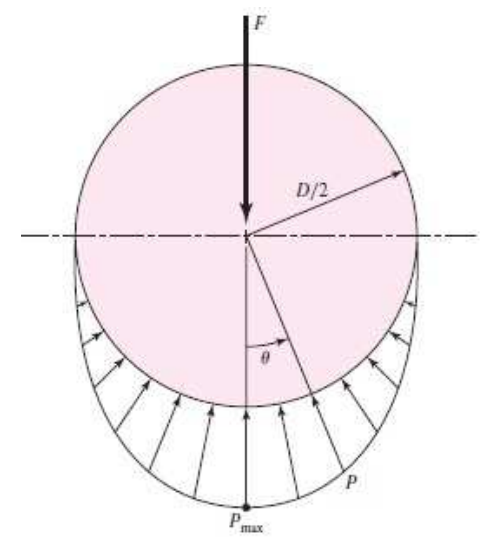

Figure 2. Pressure distribution due to axle loads on the bushing [Budynas and Nisbett(2008)]

This load imposes a friction load against the interference fit retention load, since depending on the friction coefficient between the axle and the bushing, part of the imposed load $F$ will be transferred to the bushing-housing coupling. In this text, this load is called Force Load Friction and, for $\theta_{i}=\theta_{f}=90^{\circ}$, has the equation derived below.

Knowing that

$$
p=P_{\max } \cos \theta
$$

The vertical component of $p d A$ is:

$$
p d A \cos \theta=p L\left(\frac{D}{2}\right) d \theta \cos \theta=P_{\max }\left(\frac{D L}{2}\right) \cos \theta^{2} d \theta
$$


Integrating from $\theta=-\pi / 2$ to $\theta=\pi / 2$ yields $\mathrm{F}$ :

$$
\begin{gathered}
F=\int_{-\pi / 2}^{\pi / 2} P_{\max }\left(\frac{D L}{2}\right) \cos \theta^{2} d \theta \\
P_{\max }=\frac{4 F_{x}}{\pi D L}
\end{gathered}
$$

This equation may be used just for $\theta_{i}=\theta_{f}=90^{\circ}$. Variations of this angle may be treated as shown in the following sections. Once $P_{\max }$ is known, it is possible to get the press fit friction load $F_{\text {friction. }}$. Thus,

$$
\begin{aligned}
F_{\text {friction }} & =\mu_{i n} N \\
& =\mu_{i n} \int_{A} p(\theta) d A \\
& =\mu_{i n} L R \int_{-\pi / 2}^{\pi / 2} P_{\max } \cos \theta d \theta \\
& =\mu_{i n} L R P_{\max }[\sin (\pi / 2)-\sin (-\pi / 2)] \\
& =2 \mu_{i n} L R P_{\max } \\
& =\mu_{i n} D L \frac{4 F}{\pi D L} \\
& ==\frac{4}{\pi}\left(\mu_{i n} F\right)
\end{aligned}
$$

Therefore:

$$
F_{\text {friction }}=\frac{4}{\pi}\left(\mu_{i n} F\right)
$$

This load has the directions tangential or axial, depending on the loads imposed to the bushing. It works as an adherence between the bushing and the axle, so that if $F_{\text {friction }}>$ $F_{\text {retention }}$, the bushing will migrate.

\subsection{Limitations of the theory}

Even with the analytical formulation being an established method, this methodology has limitations mainly when the load imposed against the bushing tends to be assymmetrical or if the axle is subjected to bending. Figure 3 below summarizes the applicability of the method:

\section{FINITE ELEMENT ANALYSIS}

The friction force is the reason why the bushing migration happens. That is, by definition, the friction force acting on the inner bushing surface and appearing whenever a load acts on the structure, forcing the bushing which is located between the pin (or a sleeve bushing) and the housing of the lug.

One might say that for the analysis of bushing migration, it would be necessary only the simple comparison between this friction force and the retention force (already discussed), 


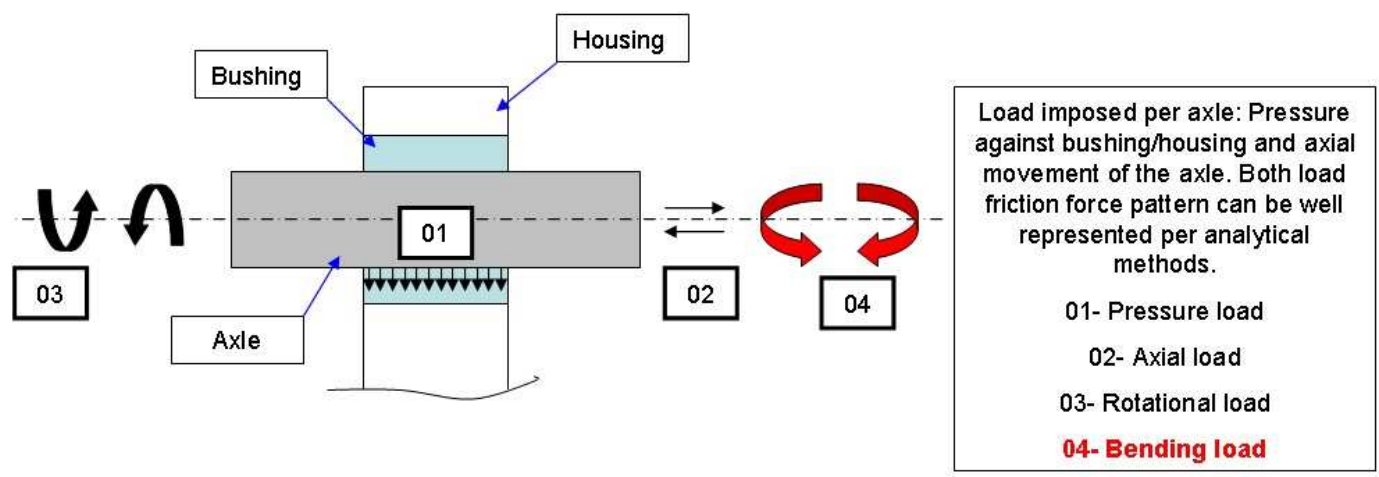

Figure 3. Analytical method limitations

once the former is greater than the later, migration occurs. But care must be taken: the retention force is affected by the pin load, depending on its intensity. The effect of the pin load is demonstrated and discussed in this section.

For that, a hypothetical case of a typical lug section (Figure 4) is studied to determine the influence of $F_{x}$ (Force in $x$ direction) on the retention force.

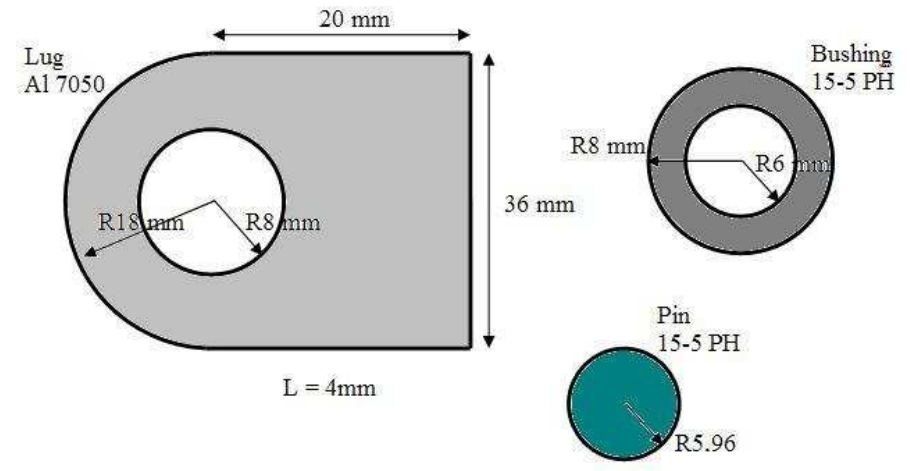

Figure 4. Hypothetical lug and bushing dimensions and properties

Figure 4 shows the dimensions and properties of a typical lug assembly. The used coordinate system and the force $F_{x}$ are shown in Figure 5. For this analysis, the hypothetical lug is fixed on its edges as in Figure 5.

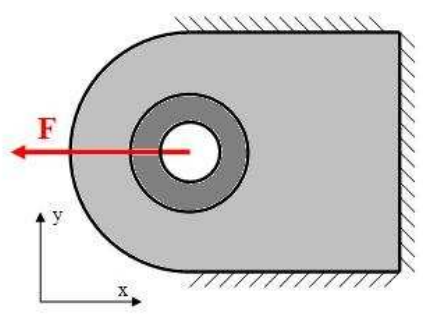

Figure 5. Hypothetical lug boundary conditions

In real cases, the $F_{x}$ force represents a load acting on plane XY. This load generates a pressure distribution along the contacting surfaces of the pin and the bushing, and the integral of this pressure on the surface gives the normal force $N$, which multiplied by the coefficient of friction of the coupling surface gives the friction force (Equation 4, or Equation 7). 
In the same manner, this load generates a pressure distribution along the contacting surfaces between bushing and lug. The difference here is that, once there is interference on this assembly, the pressure distribution already existent will be modified. The combination of the pressure distribution due to the interference and the loading will determine the new retention force $F_{\text {retention }}$.

The mathematical determination of this equation comes from the theory of pressure distribution on bushings under loaded pins, presented in section 3.2.

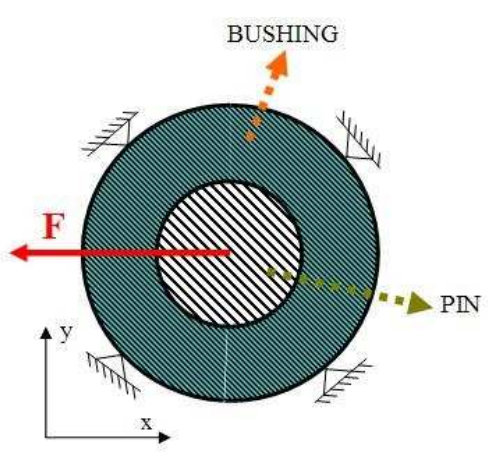

Figure 6. Pinned bushing

To validate the theory, a comparison between numerical results and the analytical equations is performed. The numerical analysis is performed with the finite element software MSC MARC. MARC is a FEM software widely used in the aerospace industry and is suitable for highly non-linear problems involving material non-linearities, buckling, geometrical non-linearities and contact. In this work the contact algorithms of MARC were specially usefull. It applies a penalty method optimization known as Augmented Lagrangean Method. For more details, see [MSC Software (Firm : Santa Ana(2011a)]. In the next sections, the results of two numerical cases are being presented. Each case is compared to the analytical solutions obtained above.

The first case represents the above mentioned hypothetical case, except that in this case the bushing has its outside surface pinned, as shown in the Figure 6. The second case is the response of the hypothetical lug already detailed.

\subsection{Finite Element Model Details}

The finite element model for the two cases was developed in MSC PATRAN (see [MSC Software (Firm : Santa Ana(2011b)]), using parabolic HEXA element formulation [Bathe(1996)] (Figure 7 and Figure 8). 


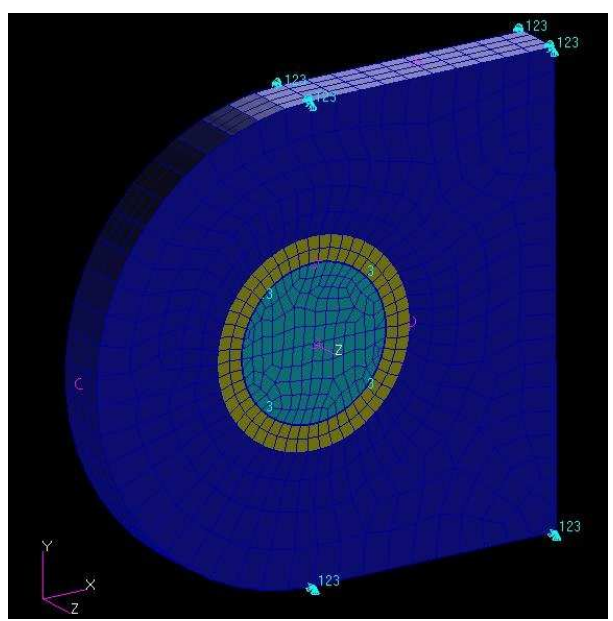

Figure 7. Hypothetical lug finite element model

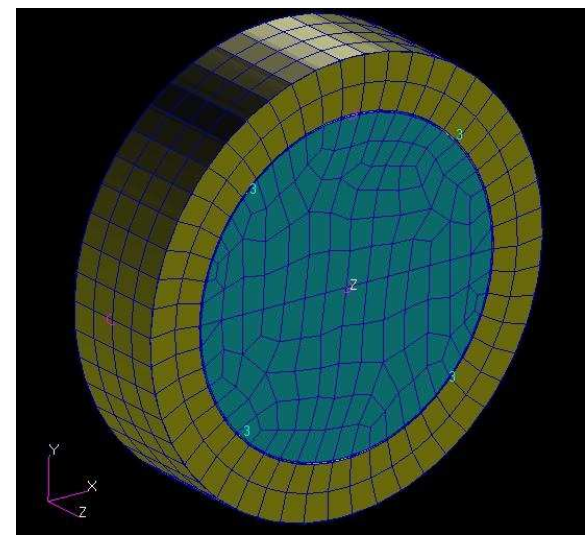

Figure 8. Pinned lug finite element model

\subsection{Pinned Bushing}

Figure 9 depicts the contact pressure distribution between pin and bushing. As it is possible to see, the area of contact grows from one simulation step to another as the force increments are added. That is, the higher the load, the higher the contact area. For this reason, it is necessary an analytical recalculation for the different initial and final angles $\left(\theta_{i}\right.$ and $\left.\theta_{f}\right)$ of this contact surface.

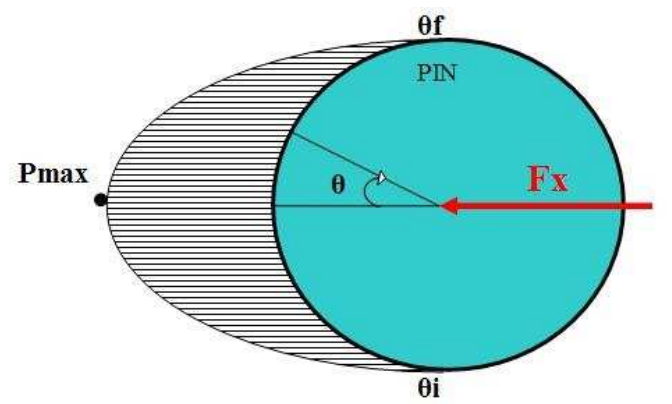

Figure 9. Contact pressure distribution 
The equation for the contact pressure varying with the contact angle becomes:

$$
p=P_{\max } \cos \left[\theta\left(\frac{\pi}{\theta_{f}-\theta_{i}}\right)\right]=P_{\max } \cos \theta^{\prime}
$$

with

$$
\theta_{i} \leq \theta \leq \theta_{f}
$$

The horizontal component of $p d A$ is:

$$
(p d A) \cos \theta=\left[p L\left(\frac{D}{2}\right) d \theta\right] \cos \theta=P_{\max }\left(\frac{D L}{2}\right) \cos \theta \cos \theta^{\prime} d \theta
$$

Integrating this from $\theta=\theta_{i}$ to $\theta_{f}$ yields $F_{x}$. Thus,

$$
\begin{aligned}
F_{x} & =\int_{\theta_{i}}^{\theta_{f}} P_{\max }\left(\frac{D L}{2}\right) \cos \theta \cos \theta^{\prime} d \theta \\
& =P_{\max }\left(\frac{D L}{2}\right) \int_{\theta_{i}}^{\theta_{f}} \cos \theta \cos \left[\theta\left(\frac{\pi}{\theta_{f}-\theta_{i}}\right)\right] d \theta
\end{aligned}
$$

for $k=\left(\frac{\pi}{\theta_{f}-\theta_{i}}\right)$

$$
\begin{aligned}
F_{x} & =\left.P_{\max }\left(\frac{D L}{2}\right)\left\{\frac{\sin [\theta(1-k)]}{2(1-k)}+\frac{\sin [\theta(1+k)]}{2(1+k)}\right\}\right|_{\theta_{i}} ^{\theta_{f}} \\
P_{\text {max }} & =\frac{F_{x}}{\left.\left(\frac{D L}{2}\right)\left\{\frac{\sin [\theta(1-k)]}{2(1-k)}+\frac{\sin [\theta(1+k)]}{2(1+k)}\right\}\right|_{\theta_{i}} ^{\theta_{f}}}
\end{aligned}
$$

For solutions of trigonometric integrals see [Abramowitz and Stegun(1964)]. Once $P_{\max }$ is known it is possible to get to $F_{\text {friction }}$ :

$$
\begin{aligned}
F_{\text {friction }} & =\mu_{i n} N \\
& =\mu i n \int_{A} p(\theta) d A \\
& =\mu_{i n} L R \int_{\theta_{i}}^{\theta_{f}} P_{\max } \cos \theta k d \theta \\
& =\left.\mu_{i n} L R P_{\max }\left\{\frac{\sin (\theta k)}{k}\right\}\right|_{\theta_{i}} ^{\theta_{f}} \\
F_{\text {friction }} & =\mu_{i n} \frac{\left.F_{x}\left\{\frac{\sin (\theta k)}{k}\right\}\right|_{\theta_{i}} ^{\theta_{f}}}{\left.\left\{\frac{\sin [\theta(1-k)]}{2(1-k)}+\frac{\sin [\theta(1+k)]}{2(1+k)}\right\}\right|_{\theta_{i}} ^{\theta_{f}}}
\end{aligned}
$$

This formulation allows for the analytical calculation of the friction force between pin and bushing. It is important too in the calculation of the retention force under loaded conditions. 
This analysis leads to closer results compared with the theoretical approach, once it better represents a cylinder inside a loaded pin.

Simulations were performed for two load values: $F_{x}=1000 \mathrm{daN}$ and $F_{x}=2400 \mathrm{daN}$. The results are shown in the following sections:

\subsection{1 $F_{x}=1000 d a N$}

The numerical analysis gives, as shown in figure 10 the initial and final angles of contact of $\theta_{i}=-\theta_{f}=39.6^{\circ}$. From these values and knowing $F_{x}, D$ and $\mathrm{L}$ it is possible to calculate Equation 4. Therefore, the anlytical value for the maximum pressure along the distribution is:

$$
P_{\text {max }}=500.54 M P a \text {. }
$$

Also, applying Equation (7), with the coefficent of friction between bushing and pin of $\mu=0.74$ (steel with steel) leads to:

$$
F_{\text {friction }}=774.47 \mathrm{daN} \text {. }
$$

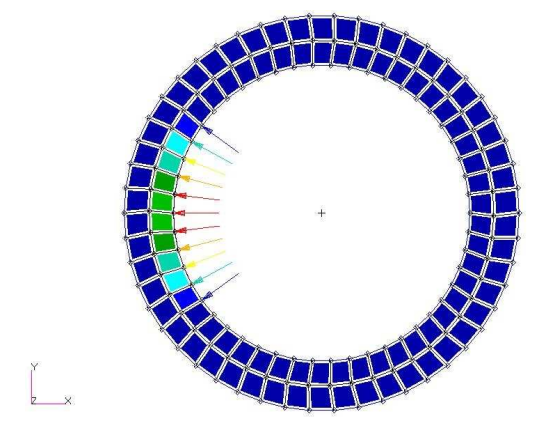

Figure 10. Contact force normal vector representation $\left(F_{x}=1000 d a N\right.$, Pinned Bushing)

The numerical simulation using MSC MARC (see [MSC Software (Firm : Santa Ana(2011a)]) gives as output the values for the contact normal forces between bushing and pin nodes of Figure 10 .The sum of the force values gives the total normal force between the two bodies. Multiplying $N \mu_{i n}$ yields the numerical result for $F_{\text {friction }}$. The pressure distribution can also be determined with the numerical results simply by summing the normal force of each row of nodes with same angle (considering a cylindrical coordinate system with origin in the center of the bushing) and dividing it by the row correspondent area $A_{\text {row }}=\pi D L / N_{\text {nodes }}$, where $N_{\text {nodes }}$ is the number of nodes around the bushing perimeter. In the simulations, $N_{\text {nodes }}=50$. Applying the described method leads to:

$$
\begin{aligned}
& P_{\text {max fem }}=485.87 \mathrm{MPa} \\
& F_{\text {friction fem }}=771.30 \mathrm{daN}
\end{aligned}
$$




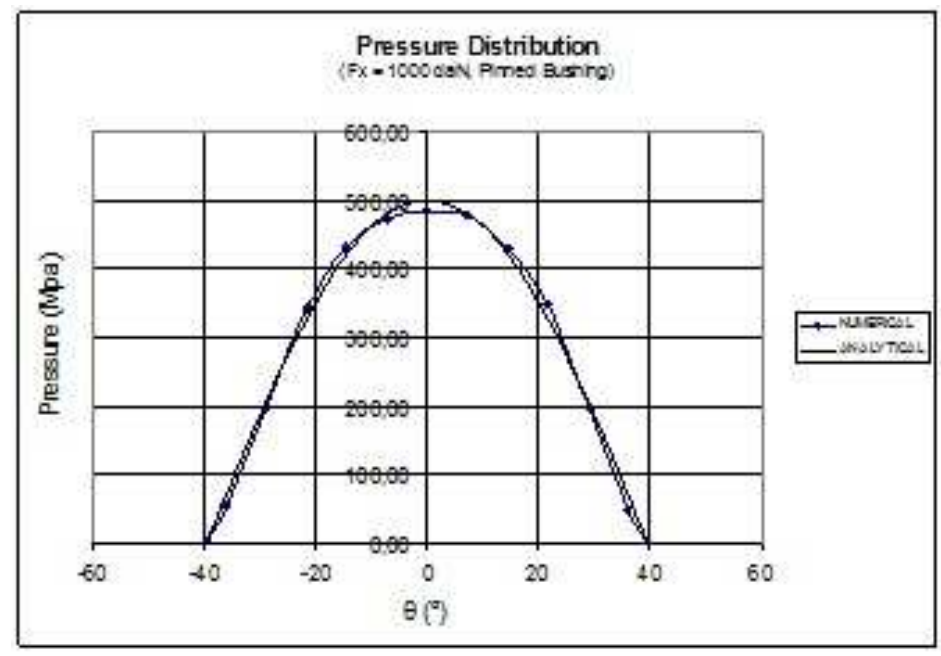

Figure 11. Pressure distribution $\left(F_{x}=1000 d a N\right.$, Pinned Bushing

Figure 11 shows the pressure distribution for both analytical and numerical analyses:

The comparison of the analytical and numerical analyses show that the error is quite low:

$$
\begin{gathered}
\operatorname{error}\left(P_{\text {max }}\right)=\frac{P_{\text {max }}}{P_{\text {max fem }}}-1=3.0 \% \\
\operatorname{error}\left(F_{\text {friction }}\right)=\frac{F_{\text {friction }}}{F_{\text {friction fem }}}-1=0.4 \%
\end{gathered}
$$

\subsection{2 $\quad F_{x}=2400 d a N$}

Figure 12 shows the initial and final angles of contact for a $F_{x}=2400 \mathrm{daN}$ load on the pin. The contact angles are $\theta_{i}=-\theta_{f}=50.4^{\circ}$. With these angles and using the analytical equations, the results for the maximum pressure and friction force are:

$$
\begin{gathered}
P_{\text {max }}=971.17 \mathrm{MPa} \\
F_{\text {friction }}=1912.46 \mathrm{daN}
\end{gathered}
$$

And the numerical results are:

$$
\begin{aligned}
& P_{\text {max fem }}=907.15 \mathrm{MPa} \\
& F_{\text {friction fem }}=1908 \mathrm{daN}
\end{aligned}
$$

Figure 13 shows the pressure distribution along the bushing contact surface for both the analytical and the numerical analyses.

The comparison of the obtained results gives: 


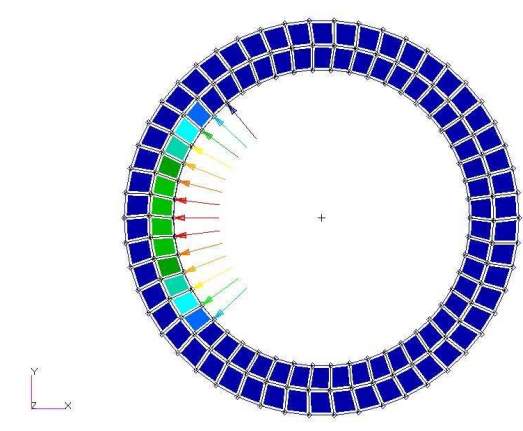

Figure 12. Contact Force normal vector representation $\left(F_{x}=2400 d a N\right.$, Pinned Bushing $)$

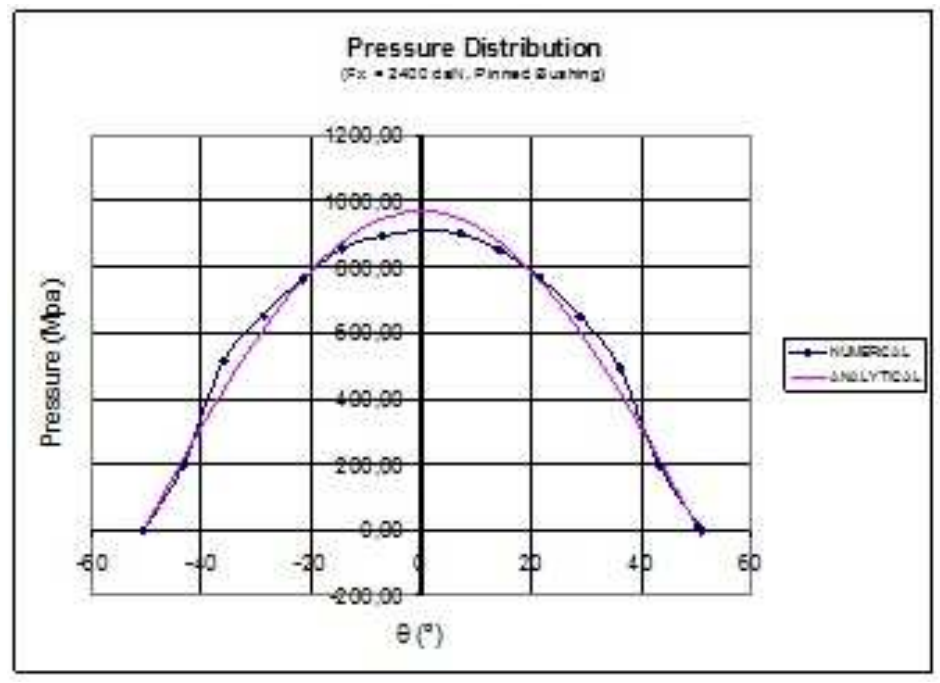

Figure 13. Pressure distribution $\left(F_{x}=2400 d a N\right.$, Pinned Bushing

$$
\begin{gathered}
\operatorname{error}\left(P_{\text {max }}\right)=\frac{P_{\text {max }}}{P_{\text {max fem }}}-1=7.0 \% \\
\operatorname{error}\left(F_{\text {friction }}\right)=\frac{F_{\text {friction }}}{F_{\text {friction fem }}}-1=0.2 \%
\end{gathered}
$$

\subsection{Hypothetical Lug Assembly}

In the previous sections, it was discussed the influence on the pressure distribution on the interface between pin and bushing due to the application of load on the pin. In the same manner, this load generates a pressure distribution along the contacting surfaces between bushing and lug. The difference here is that, once there is interference on this assembly, the pressure distribution already existent will be modified. The combination of the pressure distribution due to interference and loading will determine the new retention force $F_{\text {retention }}$.

In this new analysis, an interference of $0,032 \mathrm{~mm}$ on lug/bushing assembly is being considered. It represents an arbitrary value within the H6s6 tolerance level.

The simulation now considers the application of $F_{x}$ by a rigid RBE3 element, which makes the solution more simple and is commonly used in engineering problems. Figure 14 depicts the used model. 


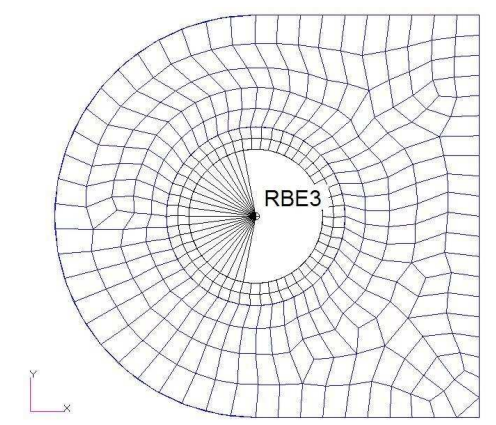

Figure 14. Application of load thru RBE3 element

Considering multiple values of $F_{x}=0 ; 200 ; 400 ; 600$ and $800 \mathrm{da} N$ the following pressure distributions are shown in Figure 15

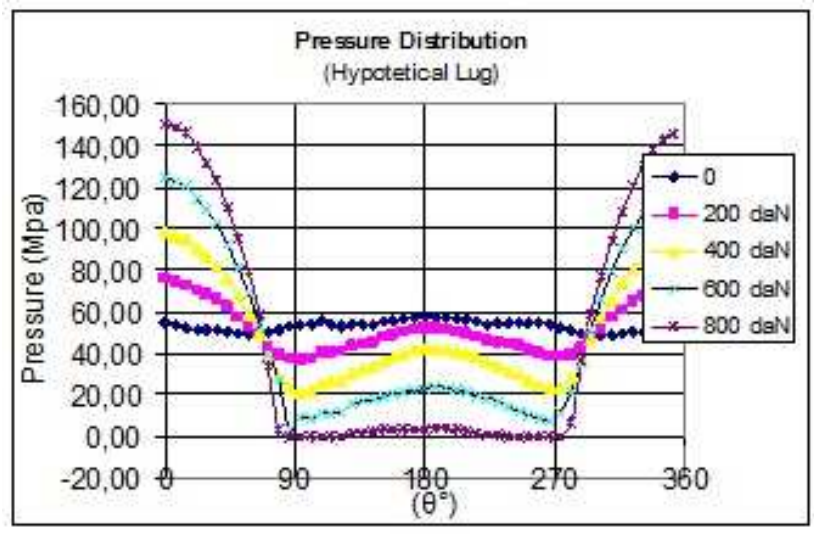

Figure 15. Pressure distribution on lug $\left(F_{x}=0 ; 200 ; 400 ; 600\right.$ and $800 d a N$

It is possible to visualize from Figure 15 that all the distribution curves tend to span through same initial and final angles. That happens because the RBE3 element angles do not vary making the pressure distribution initial and final angles constant. In this specific case, the angle values are $\theta_{i}=-\theta_{f}=88^{\circ}$.

With that in mind, it is now possible to calculate $P_{\text {max }}$ for each load case and calculate the friction force $F_{\text {friction }}$ using the analytical method already presented. For the numerical solution, the same method presented in the previous section is used, that is, the numerical simulation using MSC MARC [MSC Software (Firm : Santa Ana(2011a)] gives as output the values for the contact normal forces between the bushing and lug nodes. The sum of these forces gives the total normal force between the two bodies. Multiplying $N \mu_{\text {out }}$ (using the friction coefficient between the lug and the bushing outside surface) gives the numerical result for the retention force $F_{\text {retention }}$ and the effect of the applied load being already considered. The pressure distribution can also be determined (Figure 15) using the sum of normal forces for each row of nodes with same angle and dividing by the row correspondent area as before.

\subsubsection{Load Correction Factor}

The load application with RBE3 element does not represent with accuracy the existence of a real pin load on the bushing. Therefore, a correction factor must be applied. Using 
Equation 7 yields:

$$
F_{x}=\left.P_{\max }\left(\frac{D L}{2}\right)\left\{\frac{\sin [\theta(1-k)]}{2(1-k)}+\frac{\sin [\theta(1+k)]}{2(1+k)}\right\}\right|_{\theta_{i}} ^{\theta_{f}}
$$

Numerical results give $P_{\text {max }}$, the remaining of the equation is known from the lug dimensions. The $\theta_{i}$ and $\theta_{f}$ angles are constant as already discussed. Thus, it is possible to calculate $F_{x}$ for each load case.

The load correction factor is the direct relation between the force $F_{x}$ and the force applied by the RBE3 element:

$$
f_{\text {correction }}=\frac{F_{x}}{F_{R B E 3}}
$$

\subsubsection{Analytical curve - Ideal Lug}

The analytical response for the retention force can be calculated based on the theory presented in section 3.1 superposed to the friction force of the external bushing surface, according to the theory presented in section 4.Actually this method is a superposition of effects. In summary, the press fitted bushing can act in two different ways. Knowing that the friction force of the outside bushing surface is:

$$
F_{\text {friction }}=\mu_{\text {out }} \frac{\left.f_{\text {correction }} F_{R B E 3} \frac{\sin \theta k}{k}\right|_{\theta_{i}} ^{\theta_{f}}}{\left.\left\{\frac{\sin [\theta(1-k)]}{2(1-k)}+\frac{\sin [\theta(1+k)]}{2(1+k)}\right\}\right|_{\theta_{i}} ^{\theta_{f}}}
$$

1. Retention force is constant (no load influence)

If $F_{\text {friction }}<\mu_{\text {out }} \pi D L p$ then

$F_{\text {retention }}=\mu_{\text {out }} \pi D L p$ caused inly by interference.

2. Retention force depends on the applied load

If $F_{\text {friction }}>\mu_{\text {out }} \pi D L p$ then

$F_{\text {retention }}=F_{\text {friction }}$

Figure 16 below shows the results for the numerical solution and the ideal analytical solution for the hypothetical lug

From figure 16, it is possible to see that the retention force $F_{\text {retention }}$ is actually a little higher in the unloaded $\left(F_{x}=0\right)$ typical lug. The reason for this is obvious: since the typical lug is actually stronger than the ideal lug, it generates a higher amount of pressure in the bushing external surface for the same value of interference. Once the load gets higher, the retention force tends to decreasse, reaching the value presented by the ideal lug. Therefore, the opposite side of the bushing (not reached by the load) tends to lose contact with the lug internal surface, and the interference it once had now is applied on the side reached by the load. Thus, when this contact is completely lost, the interference doubles on the other side, generating a retention force equivalent to that on the ideal lug. 


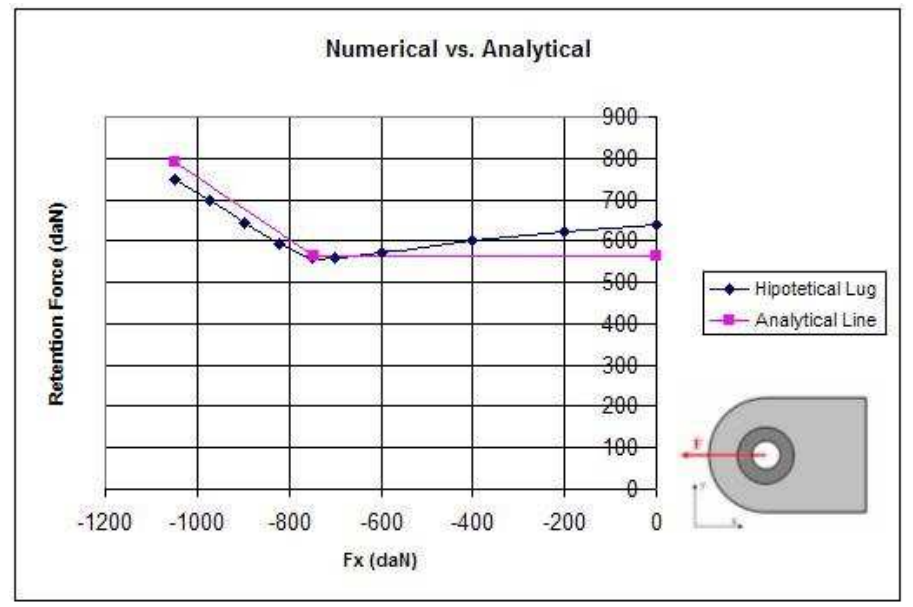

Figure 16. Retention force curve

\section{MIGRATION CRITERIA}

Once the following items are known:

- Bushing/Lug/Pin assembly properties and simensions;

- Interference fit tolerances and adjustment;

- Limit loads involved;

- Bushing outer and inner surfaces' contact friction coefficients;

is it possible to draw a diagram as in figure 17 to describe the criteria for bushing migration:

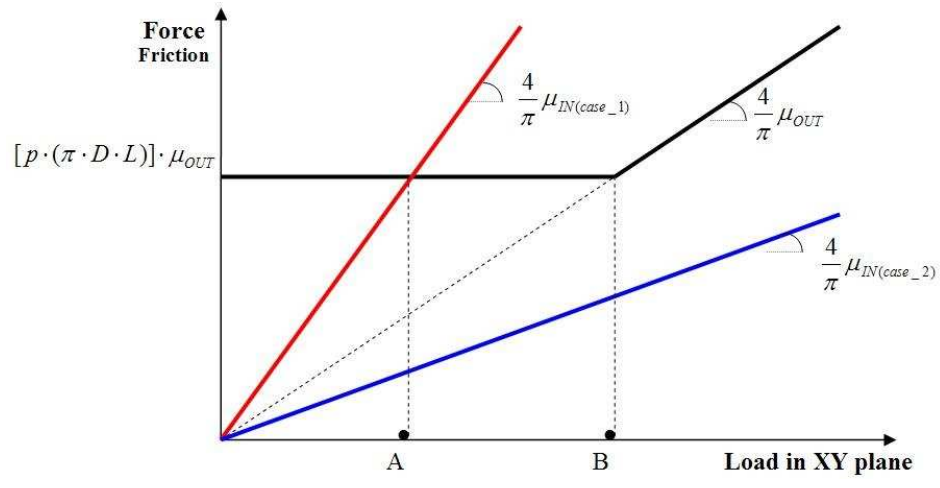

Figure 17. Bushing migration criteria diagram

Note that for a conservative design, the diagram considers a pressure distribution starting and ending with $\theta_{i}=\theta_{f}=90^{\circ}$. The black line represents the retention force on bushing due to application of load in the XY plane. As discussed earlier, this curve is described by the equations in section 4.3.2. Point B represents the transition, i.e., when the retention force is influenced by the load applied in the XY plane:

$$
B \Rightarrow p(\pi D L) \mu_{\text {out }}=F_{X Y} \frac{4}{\pi} \mu_{\text {out }}
$$


thus,

$$
F_{X Y}=\left[p\left(\pi^{2} / 4\right) D L\right]
$$

The red line (case 1) occurs when the friction coefficient acting on the inner surface of the bushing is equal or higher than the friction coefficient acting on the outer surface $\mu_{\text {in }} \geq \mu_{\text {out }}$. For this case, when the load in the XY plane is higher than that on point A, the friction force inside the bushing overcomes the friction force on the outside surface making the bushing migrate:

$$
A \Rightarrow p(\pi D L) \mu_{\text {out }}=F_{X Y} \frac{4}{\pi} \mu_{\text {in }}
$$

thus,

$$
F_{X Y}=\left[p\left(\pi^{2} / 4\right) D L\right] \frac{\mu_{\text {out }}}{\mu_{\text {in }}}
$$

The blue line (case 2) occurs when the friction coefficient on the inner surface of the bushing is smaller than the friction coefficient on the outer surface $\mu_{\text {in }}<\mu_{\text {out }}$. In this case, the friction force inside the bushing never overcomes the friction force on the outer surface, not allowing the bushing migration.

\section{SUMMARY AND CONCLUSIONS}

The present work intends to demonstrate the analytical/numerical evaluation of the bushing migration phenomena in mechanical assemblies. Based on the analyses, it is possible to conclude that the methodology can be applied to general mechanical assemblies, such as landing gear fittings, side-stay fittings, control surfaces fittings and a series of other appli-

cations. As a future extension of this work, bench tests should be performed and compared to the analytical and numerical analyses. The tests should take into account both axial and tangential load directions as in the analysis presented in this article. Another suggestion for further analyses is the use of bench results also under torsional and bending loads.

\section{ACKNOWLEDGEMENTS}

The author's would like to thank EMBRAER S.A. fot the financial support.

\section{REFERENCES}

Abramowitz, M. and Stegun, I., 1964. Handbook of Mathematical Functions: With Formulas, Graphs, and Mathematical Tables. Applied mathematics series. Dover Publications. ISBN 9780486612720.

Ashby, M. and Jones, D., 2011. Engineering Materials 1: An Introduction to Properties, Applications and Design. Elsevier Science. ISBN 9780080966663.

Bathe, K., 1996. Finite element procedures. Prentice Hall. ISBN 9780133014587.

Bruhn, E., 1973. Analysis and design of flight vehicle structures. S.R. Jacobs. 
Budynas, R. and Nisbett, J., 2008. Shigley's mechanical engineering design. Number v. 10 in McGraw-Hill series in mechanical engineering. McGraw-Hill. ISBN 9780071257633.

MSC Software (Firm : Santa Ana, C.S.P.D., 2011a. MARC: User's Guide. MSC Software, Software Products Division.

MSC Software (Firm : Santa Ana, C.S.P.D., 2011b. PATRAN: User's Guide. MSC Software, Software Products Division. 